\title{
Multi-dimensional spectrum analysis for 2-D vector velocity estimation
}

\author{
Oddershede, Niels; Løvstakken, Lasse; Torp, Hans; Jensen, Jørgen Arendt
}

Published in:

IEEE Ultrasonics Symposium

Link to article, DOI:

10.1109/ULTSYM.2007.612

Publication date:

2007

Document Version

Publisher's PDF, also known as Version of record

Link back to DTU Orbit

Citation $(A P A)$ :

Oddershede, N., Løvstakken, L., Torp, H., \& Jensen, J. A. (2007). Multi-dimensional spectrum analysis for 2-D vector velocity estimation. In IEEE Ultrasonics Symposium (pp. 2433-2437). IEEE.

https://doi.org/10.1109/ULTSYM.2007.612

\section{General rights}

Copyright and moral rights for the publications made accessible in the public portal are retained by the authors and/or other copyright owners and it is a condition of accessing publications that users recognise and abide by the legal requirements associated with these rights.

- Users may download and print one copy of any publication from the public portal for the purpose of private study or research.

- You may not further distribute the material or use it for any profit-making activity or commercial gain

- You may freely distribute the URL identifying the publication in the public portal

If you believe that this document breaches copyright please contact us providing details, and we will remove access to the work immediately and investigate your claim. 


\title{
Multi-dimensional spectrum analysis for 2-D vector velocity estimation
}

\author{
Niels Oddershede ${ }^{1}$, Lasse Løvstakken ${ }^{2}$, Hans Torp ${ }^{2}$, and Jørgen Arendt Jensen ${ }^{1}$ \\ 1) Center for Fast Ultrasound Imaging, Ørsted•DTU, Build. 349, \\ Technical University of Denmark, DK-2800 Lyngby, Denmark \\ 2) Department of Circulation and Medical Imaging, \\ Norwegian University of Science and Technology, Trondheim, Norway
}

\begin{abstract}
Wilson (1991) presented a wide-band estimator for axial blood flow velocity estimation through the use of the two-dimensional (2-D) Fourier transform. It was shown how a single velocity component was concentrated along a line in the 2-D Fourier space, where the slope was given by the axial velocity. This paper presents an expansion of this study. If data are sampled within a region, instead of along a line, a threedimensional (3-D) data matrix is created along lateral space, axial space, and pulse repetitions. It is shown, that a single velocity component will be concentrated along a plane in the 3-D Fourier space, which is found through the 3-D Fourier transform of the data matrix, and that the plane is tilted according to the axial and lateral velocity components.

Two estimators are derived for finding the plane in the 3-D Fourier space, where the integrated power spectrum is largest. The first uses the 3-D Fourier transform to find the power spectrum, while the second uses a minimum variance approach. Based on this plane, the axial and lateral velocity components are estimated. A number of phantom flow measurements, for flow-to-beam angles of 60,75 , and 90 degrees, were performed to test the estimator. The data were collected using our RASMUS experimental ultrasound scanner and a 128 element commercial linear array transducer. The receive apodization function was manipulated, creating an oscillation in the lateral direction, and multiple parallel lines were beamformed simultaneously. The two estimators were then applied to the data. Finally, an in-vivo scan of the common carotid artery was performed.

The average standard deviation was found across the phantom tube, for both the axial and the lateral velocity estimate. Twenty independent estimates were made for each positions. The average standard deviation of the lateral velocity estimates ranged from $16.4 \%$ to $2.1 \%$, relative to the peak velocity, while the average standard deviation of the axial velocity ranged from $2.0 \%$ to 0.2 $\%$. Both estimators performed best for flow-to-beam angles of 90 degrees. The in-vivo scan showed the potential of the method, yielding an estimate of the velocity magnitude independent of vessel orientation.
\end{abstract}

\section{INTRODUCTION}

Blood flow velocity estimation in ultrasound systems is usually performed by repeating a number $N_{t}$ of focused emissions along the same direction at the pulse repetition frequency $f_{p r f}$. The received signal is sampled along the beam direction at a temporal sampling frequency of $f_{s}$, corresponding to a spatial sampling in depth of $2 f_{s} / c$, where $c$ is the speed of sound. A data matrix $s(z, t)$ can then be formed with $N_{z}$ depth samples and $N_{t}$ pulse repetition samples, also known as slow-time samples.
Numerous estimators have been proposed for finding the axial velocity component from this data matrix. Kasai and coworkers [1] proposed the autocorrelation estimator, where the axial velocity is found through the average phase shift along pulse repetitions. Bonnefous and Pesque proposed a cross-correlation estimator [2], where the consecutive columns of $s(z, t)$ are cross-correlated to find the spatial shift and thus the velocity. Ferrara and Algazi [3] proposed a maximum likelihood estimator, essentially using both the envelope information and the phase information in the estimator. Wilson [4] and Torp and Kristoffersen [5] proposed to find the axial velocity component through the 2-D Fourier transform of the matrix $s(z, t)$. If only a single velocity is present within the observation window, the 2-D power spectrum will peak along a line in the spectral domain. This line was denoted an isovelocity line in [5]. The axial velocity can be estimated by finding the slope of this line [4].

The purpose of this paper is to extend the estimator presented by Wilson [4] and Torp and Kristoffersen [5] to search for both the axial and the lateral component. If data are sampled in a region both axially and laterally over a number of repetitions, a 3-D signal matrix $s(x, z, t)$ can be created. It will be shown that for a single velocity, the 3-D power spectrum of $s(x, z, t)$ will be concentrated along a plane in the 3-D Fourier space, and two estimators will be derived. The two velocity components are estimated by summing the power spectrum along the iso-velocity plane corresponding to all combinations of the possible lateral and axial velocity components $\left(v_{x}, v_{z}\right)$ and choosing the combination that yield the maximum sum. The first estimator finds the power spectral components through a Fourier transform, while the second uses a minimum variance approach [6].

\section{THREE-DIMENSIONAL FOURIER TRANSFORM OF ULTRASONIC DATA}

Most ultrasonic velocity estimation systems do numerous transmissions along the same direction, and a data matrix $s(z, t)$ can be formed, where $z$ denotes depth and $t$ slow time. Normally both variables are discrete. Wilson showed [4] that if the flow has constant velocity within the limits of $z$, the 2-D fast Fourier transform $S\left(\zeta, f_{d}\right)$ of $s(z, t)$ will be concentrated along a line in the 2-D plane spanned by the spatial frequency 
$\zeta$ and the temporal frequency $f_{d}$, and that the slope of this line is proportional to the flow velocity.

Assuming we could sample within a plane instead of just a line, a 3-D data matrix $s(x, z, t)$ could be constructed, where $x$ is lateral position, $z$ is depth, and $t$ is slow time. Lets first assume the data are continuous in all dimensions and the observation time and space are infinitely long. The 3-D Fourier transform of this matrix is given by

$$
\begin{aligned}
F\left(\chi, \zeta, f_{d}\right)= & \int_{-\infty}^{\infty} \int_{-\infty}^{\infty} \int_{-\infty}^{\infty} s(x, z, t) . \\
& e^{-j 2 \pi \chi x} e^{-j 2 \pi \zeta z} e^{-j 2 \pi f_{d} t} d x d z d t,
\end{aligned}
$$

where $\chi$ is the spatial frequency along the lateral direction, $\zeta$ is the spatial frequency along the axial direction, and $f_{d}$ is the slow-time frequency commonly known as the Doppler frequency.

Considering a constant velocity movement within the axiallateral plane and disregarding the change in spatial impulse response, the 3-D data matrix $s(x, z, t)$ can be approximated by a 2-D function $s_{0}(x, z)$ moving within the plane with axial velocity $v_{z}$ and lateral velocity $v_{x}$

$$
s(x, z, t)=s_{0}\left(x-v_{x} t, z-v_{z} t\right) .
$$

The 3-D Fourier transform of $s(x, z, t)$, as given in (1), is then

$$
\begin{aligned}
F\left(\chi, \zeta, f_{d}\right) & =\int_{-\infty}^{\infty} \int_{-\infty}^{\infty} \int_{-\infty}^{\infty} s_{0}\left(x-v_{x} t, z-v_{z} t\right) \\
& =\int_{-\infty}^{-j 2 \pi \chi x} e^{-j 2 \pi \zeta z} S^{-j 2 \pi f_{d} t} d x d z d t \\
& =S_{0}(\chi, \zeta) \int_{-\infty}^{\infty} e^{-j 2 \pi \chi v_{x} t} e^{-j 2 \pi \zeta v_{z} t} e^{-j 2 \pi f_{d} t} d t \\
& =S_{0}(\chi, \zeta) \delta\left(f_{d}+v_{x} \chi+v_{z} \zeta\right) t
\end{aligned}
$$

where $S_{0}(\chi, \zeta)$ is the 2-D Fourier transform of $s_{0}(x, z)$. The function in (3) is zero except for a plane intersecting origo. This plane in the $3-\mathrm{D}$ Fourier space spanned by $\left(\chi, \zeta, f_{d}\right)$ satisfies

$$
f_{d}=-v_{x} \chi-v_{z} \zeta .
$$

The 2-D spectrum $S_{0}(\chi, \zeta)$ is assumed separable, so that

$$
S_{0}(\chi, \zeta)=S_{x}(\chi) S_{z}(\zeta)
$$

where $S_{z}(\zeta)$ is the spectrum of the emitted signal, peaking at $\zeta= \pm 2 f_{0} / c$, where $f_{0}$ is the temporal center frequency of the emitted pulse, and $S_{x}(\chi)$ is the lateral spatial spectrum. By assuming a linear array of $2 M+1$ omnidirectional point sources, equally spaced at distance $d$, the lateral spectrum can be approximated by a scaled version of the apodization function [6]

$$
S_{x}(\chi)=\sum_{m=-M}^{M} w(m) \delta\left(\chi-\frac{m d}{\lambda z}\right),
$$

where $w(m)$ is the apodization function, and $\lambda=c / f_{0}$ is the axial wavelength. According to the Nyquist sampling theorem, the lateral sampling interval $\Delta x$ at a certain depth $z$ must therefore obey

$$
\Delta x<\frac{\lambda z}{2 M d},
$$

The assumptions of continuous data, infinitely long observation time and space, and constant velocity made in deriving (3) are of course not satisfied in ultrasonic velocity estimation. By limiting the observation space both laterally and axially by windowing the signal, a spectral broadening will occur in these dimensions and side-lobes will appear. The same goes for the limited observation time. Nevertheless, the threedimensional spectral content of an almost constant velocity within the observation window will mainly be concentrated along the plane indicated by (4). The sampling along space and time will result in spectral repetitions at all multiples of the sampling frequency. The spatial sampling can relatively easy be set to avoid aliasing, while aliasing might occur along the temporal direction at high velocities. Aliasing in the 3D power spectral domain will occur when $f_{d}$ in (4) exceeds $f_{p r f} / 2$.

\section{2-D VECTOR VELOCITY ESTIMATOR}

In [4], [5], the axial velocity was estimated by summing the 2-D power spectrum along iso-velocity lines, and choosing the velocity corresponding to the largest sum. In the vector velocity estimation case, both the axial and lateral velocities are sought and according to (3) a constant velocity will give rise to non-zero values along a plane in the 3-D power spectral space. The axial and lateral velocity components can be estimated by finding the plane satisfying (4), over which the sum of power spectral components is largest. The velocity components will then correspond to the angles with which the plane is tilted in the 3-D power spectral space.

\section{A. Periodogram estimator}

By assuming the velocity components $v_{x}$ and $v_{z}$ given, the Fourier components at the plane corresponding to these velocities are given by

$$
\begin{aligned}
F\left(\chi, \zeta \mid v_{x}, v_{z}\right)= & \int_{-\infty}^{\infty} \int_{-\infty}^{\infty} \int_{-\infty}^{\infty} s(x, z, t) . \\
& e^{-j 2 \pi\left[\chi\left(x-v_{x} t\right)+\zeta\left(z-v_{z} t\right)\right]} d x d z d t
\end{aligned}
$$

which is found by inserting (4) into (1). The total power along this plane is found by integrating the magnitude squared of (8) over all spatial frequencies $\chi$ and $\zeta$

$$
P\left(v_{x}, v_{z}\right)=\int_{-\infty}^{\infty} \int_{-\infty}^{\infty}\left|F\left(\chi, \zeta \mid v_{x}, v_{z}\right)\right|^{2} d \chi d \zeta .
$$

Since data are sampled both spatially and temporally, the integrals become sums and the problem can be solved in matrix form. By ordering the 3-D signal matrix in a row vector s of length $N_{x} N_{z} N_{t}$, and defining the row vector

$$
\mathbf{e}_{i}=e^{j 2 \pi\left(\chi_{i}\left(x-v_{x} t\right)+\zeta_{i}\left(z-v_{z} t\right)\right)}
$$

for spatial frequencies $\chi_{i}$ and $\zeta_{i}$, we have

$$
F\left(\chi_{i}, \zeta_{i} \mid v_{x}, v_{z}\right)=\mathbf{e}_{i}^{H} \mathbf{s},
$$


where $(\cdot)^{H}$ denotes the complex conjugate transpose. By making a $\mathbf{e}_{i}$ vector for all $I$ permutations of the spatial frequencies, the total power along the plane given by velocities $v_{x}$ and $v_{z}$ is found by

$$
\begin{aligned}
P\left(v_{x}, v_{z}\right) & =\sum_{i=0}^{I-1}\left|F\left(\chi_{i}, \zeta_{i} \mid v_{x}, v_{z}\right)\right|^{2} \\
& =\sum_{i=0}^{I-1} \mathbf{e}_{i}^{H} \mathbf{s}\left(\mathbf{e}_{i}^{H} \mathbf{s}\right)^{H}=\sum_{i=0}^{I-1} \mathbf{e}_{i}^{H} \mathbf{R} \mathbf{e}_{i} .
\end{aligned}
$$

where $\mathbf{R}=E\left\{\mathbf{s s}^{H}\right\}$ is the covariance matrix of the data, and $E\{\cdot\}$ denotes the expectation value. Estimation of the covariance matrix is considered in Section III-B. By doing the calculation in (12) for all combinations of $v_{x}$ and $v_{z}$, the velocity components can be estimated by finding the velocities yielding the highest power along the plane

$$
\begin{aligned}
& \hat{v}_{x}=\arg \max _{v_{x}} P\left(v_{x}, v_{z}\right) \\
& \hat{v}_{z}=\arg \max _{v_{z}} P\left(v_{x}, v_{z}\right) .
\end{aligned}
$$

These discrete estimates can be further refined by interpolation of the $P\left(v_{x}, v_{z}\right)$ function.

\section{B. Minimum variance estimator}

The periodogram estimator suffers from poor spectral resolution when the observation window is limited [6], as is the case here. If the signal covariance matrix $\mathbf{R}$ is known, the power spectral component at lateral frequency $\chi_{i}$ and axial frequency $\zeta_{i}$ on the plane given by $v_{x}$ and $v_{z}$ can be found by a minimum variance estimator [6]

$$
\left|F_{m v}\left(\chi_{i}, \zeta_{i} \mid v_{x}, v_{z}\right)\right|^{2}=\frac{1}{\mathbf{e}_{i}^{H} \mathbf{R}^{-1} \mathbf{e}_{i}},
$$

where $\{\cdot\}^{-1}$ denotes the matrix inverse. Again, the estimates along the plane are summed to find the total power on the plane given by $v_{x}$ and $v_{z}$. This can be found by

$$
P_{m v}\left(v_{x}, v_{z}\right)=\sum_{i=0}^{I-1} \frac{1}{\mathbf{e}_{i}^{H} \mathbf{R}^{-1} \mathbf{e}_{i}},
$$

Since the data covariance matrix is not known, an estimate must be found. This can be done by dividing the data cube $s(x, z, t)$ of size $\left[N_{x}, N_{z}, N_{t}\right]$ into sub-cubes of size $\left[M_{x}, M_{z}, M_{t}\right]$. Hereby a total of $\left(N_{x}-M_{x}+1\right)\left(N_{z}-M_{z}+\right.$ 1) $\left(N_{t}-M_{t}+1\right)$ sub-cubes can be created. This is essentially a three-dimensional form of sub-array averaging used in some adaptive beamformers [7]. By organizing each data sub-cube in a row vector $\mathbf{s}_{m x, m z, m t}$, where $m x, m z$, and $m t$ are indexes to the first sub-cube element, the covariance matrix of size $\left[M_{x} M_{z} M_{t}, M_{x} M_{z} M_{t}\right]$ can be estimated by

$$
\hat{\mathbf{R}}=\sum_{m x=0}^{N_{x}-M_{x}} \sum_{m z=0}^{N_{z}-M_{z}} \sum_{m t=0}^{N_{t}-M_{t}} \mathbf{s}_{m x, m z, m t} \mathbf{s}_{m x, m z, m t}^{H}
$$

To ensure full rank of $\hat{\mathbf{R}}$, needed for finding the inverse, the inequality $\left(N_{x}-M_{x}+1\right)\left(N_{z}-M_{z}+1\right)\left(N_{t}-M_{t}+1\right) \geq$ $M_{x} M_{z} M_{t}$ must be satisfied. The sum along the plane in the
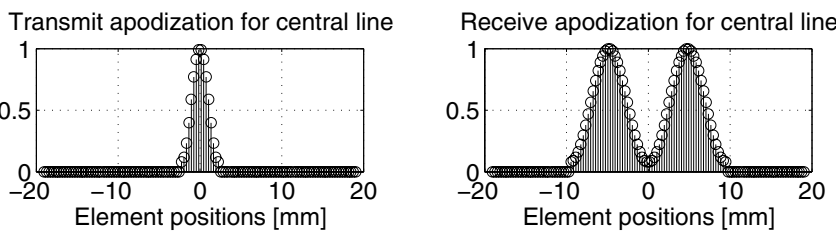

Fig. 1. Transmit and receive apodization used for central line. A narrow transmit apodization is used to create a broad beam, enabling parallel beamforming. The receive apodization creates a short broadband oscillation in the lateral direction of the point spread function.
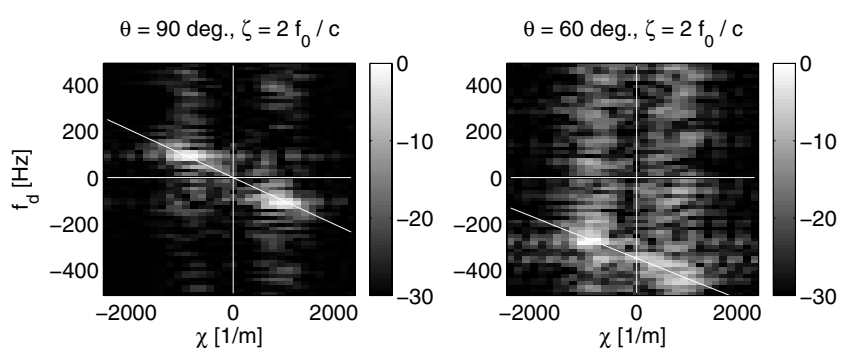

Fig. 2. Examples of power spectra at $\zeta=2 f_{0} / c$ on a dB scale. Data from phantom measurements at $\theta=90^{\circ}$ (left) and $\theta=60^{\circ}$ (right). A white line indicates $f_{d}=-v_{x} \chi-v_{z} \zeta$.

power spectral domain can then be found from (16) by creating the vectors $\mathbf{e}_{i}$ according to the new data dimensions, and using the estimated covariance matrix $\hat{\mathbf{R}}$. Again, the lateral and axial velocity estimates are found by a search for the highest total power as in (13) and (14).

\section{MEASUREMENTS}

The estimators derived in Section III search for the plane in the 3-D Fourier domain where the power is largest, but through (3) and (5) the power is limited in the $\zeta$-direction by the spectrum of the emitted wave, and in the $\chi$-direction by the apodization through (6). If a rectangular or normally windowed apodization is used, the lateral spectrum $S_{x}(\chi)$ will be centered at $\chi=0$. But by using the receive apodization shown in Fig. 1, which will create an oscillation in the lateral direction of the point spread function [8], [9], [10], the plane will intersect four clouds in the 3-D spectral domain instead of two. This is expected to increase the lateral estimator performance.

A series of measurements was performed in a flow-rig phantom. A blood mimicking fluid was circulated at a constant velocity through a tube submerged in a water tank. A linear array transducer was fixed above the tube at a controlled angle. Three measurements were made with angles of $\theta=60^{\circ}, 75^{\circ}$, and $90^{\circ}$ (transverse flow) between the transducer axis and the flow direction. The data were collected using our RASMUS experimental ultrasound scanner [11]. The parameters for the measurements are given by Table I. At every emission, $N_{x}=7$ parallel lines around the center of emission are beamformed at inter-line distance $\Delta x=200 \mu \mathrm{m}$ using delay-and-sum beamforming [6]. The lines are beamformed at $f_{s}=20 \mathrm{MHz}$, 
corresponding to an axial sampling interval of $37 \mu \mathrm{m}$. For every point along the line, where the vector velocity is estimated, $N_{z}=33$ samples along depth are used, corresponding to both a lateral and axial extent of $1.2 \mathrm{~mm} . N_{t}=32$ repetitions are made along the same direction. Clutter filtering was performed by subtracting the average of $N_{t}=32$ slow-time samples at each spatial position. The spectra of the data at $\zeta=2 f_{0} / c$ are shown in Fig. 2 for the $\theta=90^{\circ}$ (left) and $\theta=60^{\circ}$ (right) measurement. Also shown is the line corresponding to (4).

TABLE I

PARAMETERs.

\begin{tabular}{ll}
\hline Transducer & \\
\hline Transducer type & Linear array \\
Number of transducer elements & 128 \\
Transducer element pitch, $d$ & $0.3 \mathrm{~mm}$ \\
Transducer element kerf & $0.035 \mathrm{~mm}$ \\
Transducer element height & $5 \mathrm{~mm}$ \\
Elevation focus & $20 \mathrm{~mm}$ \\
Center frequency, $f_{0}$ & $5 \mathrm{MHz}$ \\
Speed of sound, $c$ & $1481 \mathrm{~m} / \mathrm{s}$ \\
Wavelength, $\lambda=c / f_{0}$ & $0.296 \mathrm{~mm}$ \\
\hline Transmission & \\
\hline Excitation type & $1.5 \mathrm{cycle}$ sinusoid at $f_{0}$ \\
Transmit apodization & $16 \mathrm{element} \mathrm{Hamming}$ \\
Receive apodization & Two 32 element Hamming \\
Transmit focus & $20 \mathrm{~mm}$ \\
Receive focus & Dynamic \\
Pulse repetition frequency, $f_{p r f}$ & $1 \mathrm{kHz}$ \\
\hline Phantom vessel & \\
\hline Vessel radius, $R$ & $6 \mathrm{~mm}$ \\
Flow angles, $\theta$ & $60^{\circ}, 75^{\circ}$ and $90^{\circ}$ \\
Depth of tube center, $z_{0}$ & 22,24, and $19 \mathrm{~mm}$, respect. \\
Peak velocity, $v_{0}$ & $10 \mathrm{~cm} / \mathrm{s}$ \\
\hline Processing & \\
\hline Lateral sampling interval & $0.2 \mathrm{~mm}$ \\
Axial sampling interval & $0.037 \mathrm{~mm}$ \\
Number of points lateral, $N_{x}$ & 7 \\
Number of points axial, $N_{z}$ & 33 \\
Number of pulses per estimate, $N_{t}$ & 32 \\
Size of region of interest, $[$ lateral, axial] & {$[1.2,1.2] \mathrm{mm}$} \\
Lateral samples in sub-cube, $M_{x}$ & 6 \\
Axial samples in sub-cube, $M_{z}$ & 6 \\
Temporal samples in sub-cube, $M_{t}$ & 12 \\
Clutter filtering method & Mean subtraction \\
Number of freq. lateral, MV, $N_{\chi}$ & 11 \\
Number of freq. axial, MV, $N_{\zeta}$ & 11 \\
Number of frequency permutations, $I$ & 121 \\
Velocity search range & $-12 \mathrm{to} 12 \mathrm{~cm} / \mathrm{s}$ \\
Velocity search interval & $0.5 \mathrm{~cm} / \mathrm{s}$ \\
\hline & \\
\hline
\end{tabular}

The two estimators were applied. $N_{\text {rep }}=18$ repetitions were made, creating 18 independent velocity profiles through the tube at each beam-to-flow angle. The average velocity profile and the standard deviation profile was found. Fig. 3 gives the results of the phantom measurement at $\theta=75^{\circ}$, showing the average profile (solid, thin line), the average plus/minus three times the standard deviation profile (dashed lines), and the expected profile (solid, thick line). The top left plot shows the lateral velocity estimates using the Periodogram estimator, while the top right plot shows the axial velocity estimates using the Periodogram estimator. The bottom left plot shows the lateral velocity estimates using the Minimum Variance estimator, while the bottom right plot shows the axial velocity estimates using the Minimum Variance estimator.
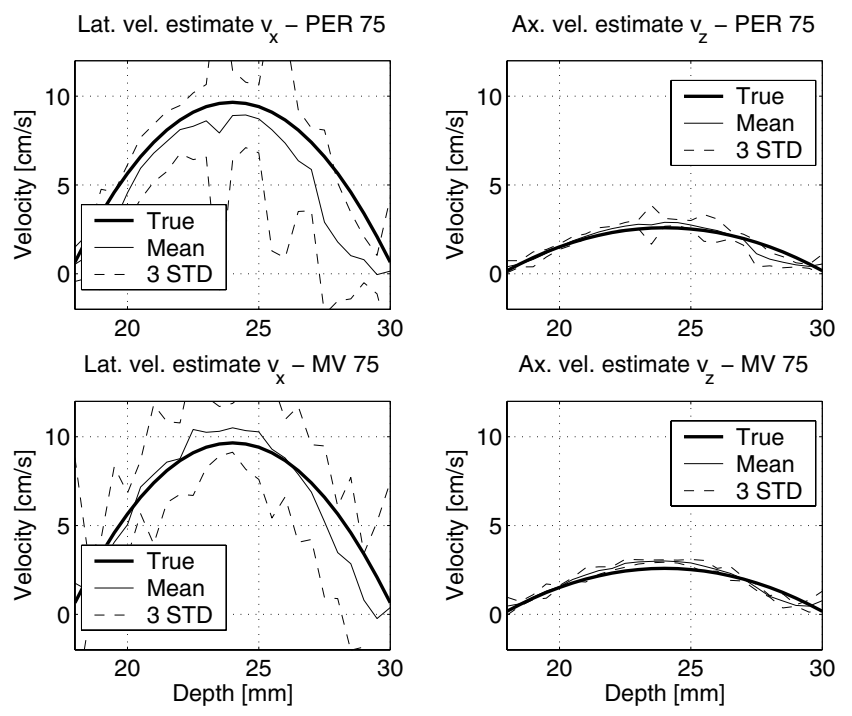

Fig. 3. Phantom measurement at a beam-to-flow angle of $\theta=75^{\circ}$. All plots give the expected profile (thick solid line), the average of 18 estimated profiles (thin solid line), and the average \pm 3 times the standard deviation (dashed line). The top row gives the results from using the Periodogram estimator, while the bottom row gives the results from the Minimum Variance estimator. The left column is the lateral velocity estimates, while the right column is the axial velocity estimates.

Fig. 3 reveals a better performance in estimating the axial velocity component (right column) than the lateral velocity component (left column). To compare the estimators across the three beam-to-flow angles, the variance and bias was averaged across the profile. Tab. II and Fig. 4 summarize the standard deviation and bias for all three beam-to-flow angles and both velocity estimators.

TABLE II

Average StANDARD DEVIATION (ASD) AND AVERAGE bias (AB)

ACROSS PROFILE, GIVEN IN \% OF PEAK VELOCITY $v_{0}=10 \mathrm{CM} / \mathrm{s}$.

RESULTS ARE GIVEN FOR BOTH THE PERIODOGRAM ESTIMATOR (PER) AND THE MINIMUM VARIANCE ESTIMATOR (MV).

\begin{tabular}{lllll}
\hline ASD & & & & \\
\hline Angle, $\theta$ & PER $v_{x}$ & PER $v_{z}$ & MV $v_{x}$ & MV $v_{z}$ \\
$60^{\circ}$ & $16.4 \%$ & $1.7 \%$ & $14.2 \%$ & $2.0 \%$ \\
$75^{\circ}$ & $11.5 \%$ & $1.1 \%$ & $11.8 \%$ & $0.7 \%$ \\
$90^{\circ}$ & $2.1 \%$ & $0.2 \%$ & $9.4 \%$ & $1.4 \%$ \\
\hline AB & & & & \\
\hline Angle, $\theta$ & PER $v_{x}$ & PER $v_{z}$ & MV $v_{x}$ & MV $v_{z}$ \\
$60^{\circ}$ & $-23.6 \%$ & $-3.4 \%$ & $-7.2 \%$ & $-1.9 \%$ \\
$75^{\circ}$ & $-14.1 \%$ & $0.1 \%$ & $-2.1 \%$ & $1.4 \%$ \\
$90^{\circ}$ & $-5.8 \%$ & $0.1 \%$ & $1.3 \%$ & $-0.3 \%$ \\
\hline
\end{tabular}

Comparing the two estimators, the average standard deviation (ASD) does not differ significantly, except for the $\theta=90^{\circ}$ experiment, where the periodogram estimator experiences a lower ASD than the minimum variance estimator. Nevertheless, the average bias of the lateral velocity estimates (bottom left) is much larger for the periodogram estimator, than for the minimum variance estimator. None of the two estimators therefore outperform the other in all cases. 

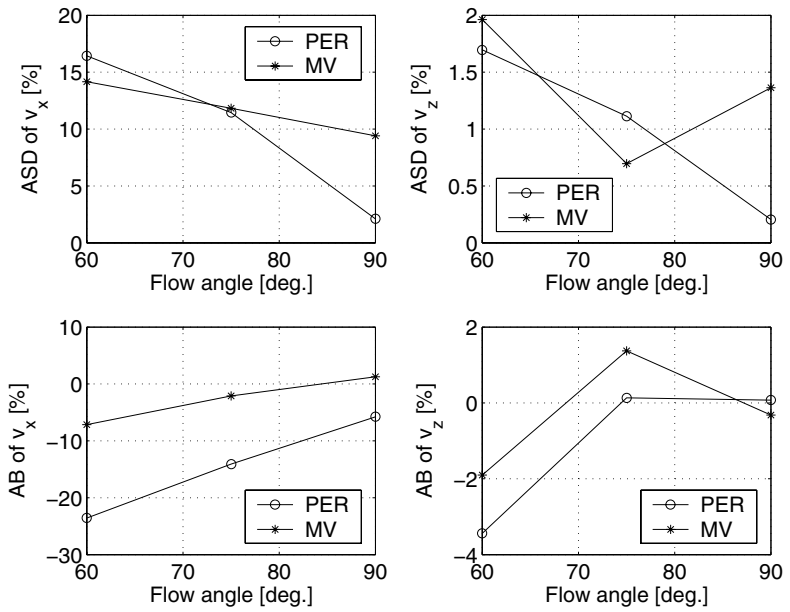

Fig. 4. Performance of the two estimators at different beam-to-flow angles, results from Table II. The top row gives the average standard deviation (ASD) for the lateral velocity estimates (left) and the axial velocity estimates (right). The bottom row gives the average bias (AB) for the lateral velocity estimates (left) and the axial velocity estimates (right). The results using the periodogram estimator is marked by a circle, while the results from the Minimum Variance estimator is marked by a star.

\section{IN-VIVO MEASUREMENT}

An in-vivo measurement has been performed using the proposed method. The common carotid artery of a healthy $32-$ year-old male volunteer was scanned for 3 seconds using our RASMUS experimental ultrasound scanner [11]. The parameters were the same as in Table I, except the pulse repetition frequency was set at $f_{p r f}=6 \mathrm{kHz}$. The vector velocities were estimated from 32 repeated emissions along 17 different lines, $1.2 \mathrm{~mm}$ apart. Along each line, the vector velocity was estimated at every $0.5 \mathrm{~mm}$ in depth. A B-mode image was created from the same data, using a single Hamming window as receive apodization. From the vector velocity estimates, the velocity magnitude $|\hat{v}|=\sqrt{\hat{v}_{x}^{2}+\hat{v}_{z}^{2}}$ was found, and a color flow map (CFM) was created showing both the B-mode image at $40 \mathrm{~dB}$ dynamic range (black and white) and the velocity magnitude from 0 to $1.2 \mathrm{~m} / \mathrm{s}$ (color). A CFM from the diastolic period is shown in Fig. 5. The left part of Fig. 5 shows the CFM created using the periodogram estimator from Section III-A, while the rightmost plot of Fig. 5 shows the CFM using the minimum variance estimator from Section IIIB. Note that no post processing of the velocity estimates has been performed.

\section{CONCLUSION}

This paper has shown that when data are sampled in a rectangular spatial window over time, a single velocity component is concentrated along a plane in the three-dimensional power spectrum spanned by lateral, axial, and temporal frequencies. The plane will be tilted according to the lateral and axial velocity components. Two estimators were derived for finding the two velocity components, the first based on the periodogram and the second on minimum variance. Both estimators were
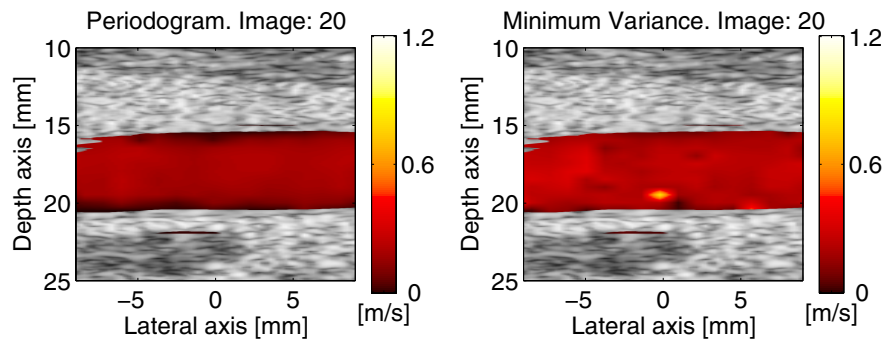

Fig. 5. Color flow map of the common carotid artery of a healthy male volunteer during diastole. The leftmost part shows the magnitude of the velocity estimates found using the periodogram estimator, while the rightmost part shows that using the minimum variance estimator.

tested on measured phantom data for beam-to-flow angles of $60^{\circ}, 75^{\circ}$, and $90^{\circ}$. The axial velocity estimates showed a standard deviation and bias an order of magnitude below that of the lateral velocity estimates, and the performance of the vector velocity estimators was increased as the beam-toflow angle approached $90^{\circ}$. An in-vivo transverse scan of the common carotid artery was performed, showing the potential of the method under in-vivo conditions.

\section{ACKNOWLEDGEMENT}

This work was supported by grant 26-04-0024 from the Danish Science Foundation and by B-K Medical, Herlev, Denmark. The authors wish to thank MD Kristoffer Lindskov Hansen, University Hospital of Copenhagen, Denmark, for performing the in-vivo scan.

\section{REFERENCES}

[1] C. Kasai, K. Namekawa, A. Koyano, and R. Omoto. Real-time twodimensional blood flow imaging using an autocorrelation technique. IEEE Trans. Son. Ultrason., 32:458-463, 1985.

[2] O. Bonnefous and P. Pesqué. Time domain formulation of pulse-Doppler ultrasound and blood velocity estimation by cross correlation. Ultrason. Imaging, 8:73-85, 1986.

[3] K. W. Ferrara and V. R. Algazi. A new wideband spread target maximum likelihood estimator for blood velocity estimation - Part I: Theory. IEEE Trans. Ultrason., Ferroelec., Freq. Contr., 38:1-16, 1991a.

[4] L. S. Wilson. Description of broad-band pulsed Doppler ultrasound processing using the two-dimensional Fourier transform. Ultrason. Imaging, 13:301-315, 1991.

[5] H. Torp and K. Kristoffersen. Velocity matched spectrum analysis: A new method for suppressing velocity ambiguity in pulsed-wave doppler. Ultrasound Med. Biol., 21(7):937-944, 1995.

[6] D. H. Johnson and D. E. Dudgeon. Array signal processing. Concepts and techniques. Prentice-Hall., Englewood Cliffs, New Jersey, 1993.

[7] H. Krim and M. Viberg. Two decades of array signal processing research. IEEE Signal Processing Magazine, 13:67-94, July 1996.

[8] J. A. Jensen and P. Munk. A new method for estimation of velocity vectors. IEEE Trans. Ultrason., Ferroelec., Freq. Contr., 45:837-851, 1998.

[9] M. E. Anderson. Spatial quadrature: a novel technique for multidimensional velocity estimation. In Proc. IEEE Ultrason. Symp., volume 45, pages 1233-1238, 1997.

[10] J. A. Jensen. A new estimator for vector velocity estimation. IEEE Trans. Ultrason., Ferroelec., Freq. Contr., 48(4):886-894, 2001.

[11] J. A. Jensen, O. Holm, L. J. Jensen, H. Bendsen, S. I. Nikolov, B. G. Tomov, P. Munk, M. Hansen, K. Salomonsen, J. Hansen, K. Gormsen, H. M. Pedersen, and K. L. Gammelmark. Ultrasound research scanner for real-time synthetic aperture image acquisition. IEEE Trans. Ultrason., Ferroelec., Freq. Contr., 52 (5):881-891, May 2005. 